\title{
A Particle-In-Cell Solver based on a High-order Hybridizable Discontinuous Galerkin Spectral Element Method on Unstructured Curved Meshes
}

\author{
M. Pfeiffer ${ }^{\mathrm{a}}$, F. Hindenlang ${ }^{\mathrm{b}}$, T. Binder ${ }^{\mathrm{a}}$, S. M. Copplestone ${ }^{\mathrm{c}}$, C.-D. Munz ${ }^{\mathrm{c}}$, S. \\ Fasoulas $^{\mathrm{a}}$ \\ ${ }^{a}$ Institute of Space Systems, University of Stuttgart, Pfaffenwaldring 29, D-70569 Stuttgart, \\ Germany \\ ${ }^{b}$ Max-Planck Institute for Plasma Physics, Boltzmannstr. 2, D-85748 Garching, Germany \\ ${ }^{c}$ Institute of Aerodynamics and Gasdynamics, University of Stuttgart, 70550 Stuttgart, \\ Germany
}

\begin{abstract}
A high-order hybridizable discontinuous Galerkin spectral element method (HDGSEM) for Particle-In-Cell (PIC) schemes is presented for the simulation of electrostatic applications on three-dimensional unstructured curved meshes. The electrostatic Poisson equation is solved and optionally a Boltzmann relation for the electron species can be used which leads to non-linear source terms. The hybridizable formulation reduces the total number of unknowns of the field solver, allowing the simulation of large problems. The implementation of the HDGSEM solver in a PIC code is described and validated using several test cases with successive increasing complexity. It is shown that the high-order convergence properties are retained on curvilinear meshes, likewise when material jumps are introduced. The simulation of an ion optic illustrates the applicability of the presented method for complex geometries and large problem sizes.
\end{abstract}

Keywords: High-order, Discontinuous Galerkin Spectral Element Method, Particle-In-Cell, Electron Fluid, Non-linear Poisson, Ion optics, hybridizable DG, curvilinear mesh,

Email addresses: mpfeiffer@irs.uni-stuttgart.de (M. Pfeiffer), florian.hindenlang@ipp.mpg.de (F. Hindenlang), binder@irs. uni-stuttgart.de (T. Binder), copplestone@iag.uni-stuttgart.de (S. M. Copplestone), munz@iag.uni-stuttgart.de (C.-D. Munz), fasoulas@irs.uni-stuttgart.de (S. Fasoulas)

Preprint submitted to Computer methods in applied mechanics and engineeringJanuary 31, 2019 


\section{Introduction}

Many plasma applications are far away from the thermal and/or chemical equilibrium or quasineutral plasma conditions, e.g., electric space propulsion systems [1], plasma instabilities [2], vacuum discharges [3] and more. Therefore, 5 it is very difficult or not possible to simulate such applications with classic continuum methods. Instead, the Boltzmann equation or the collisionless special case named Vlasov equation has to be solved in such plasma conditions. A well established way to find an approximated solution of this equations is the Particle-in-Cell (PIC) method 2, 4.

The main idea of the PIC method is the splitting of the particle movement that represents the plasma and the calculations of the electric fields produced by the particle charge and boundary conditions. The electric fields are calculated on a fixed grid in an Eulerian manner. The freely moving particles and the electric fields are coupled by two interpolation steps. In the first step, the charge information of the particles is interpolated to the fixed mesh. The electric field is calculated on the mesh using the charge information as source terms. In the second step, the new electric fields are interpolated at the particle positions, in order to compute the Lorentz force and move each particle.

The PIC approach for fully kinetic simulations including electrons in complex three-dimensional geometries requires a high spatial and temporal resolution to achieve stable and physically correct results. Even though the electrostatic assumption on the field evolution allows to exclude the time scale of electromagnetic waves, the plasma frequency still needs to be resolved. The fast electron time scale can be excluded by using the Boltzmann relation for the electron species. Spatially, the Debye length must be sufficiently resolved [2]. These requirements lead to a high computational effort. On the one hand, a huge number of simulation particles can be necessary depending on the plasma conditions. A very computational consuming part in the particle movement is the particle tracking, especially in the case of complex geometries, e.g., electric 
propulsion systems on unstructured grids in $3 \mathrm{D}$.

Compared to low order field solvers, the high order Discontinuous Galerkin method allows a sub-cell resolution and therefore keeps high accuracy of the field solver even on coarse unstructured meshes [5]. Consequently, using a coarse mesh with a high-order field solver can significantly reduce the total cost of a particle simulation, even though the particle tracking cost per cell is higher.

It will be shown that an accurate solution with curved wall boundary conditions can only be found if the coarse mesh has elements with curved element sides. Furthermore, the solver should have a good parallel performance. Otherwise it would not be possible to simulate complex 3D applications. Regarding efficiency, two choices will be made. First, we restrict ourselves to hexahedral curved elements and the discontinuous Galerkin spectral element methods [6], where integration and interpolation points are collocated, yielding sparse tensorproduct operators and diagonal mass matrices. Secondly, we apply the hybridizable approach [7, to reduce the number of unknowns involved in the Poisson solver from volume to surface degrees of freedom, which is especially beneficial in the high-order case [8, 9. We refer to the method as HDGSEM.

The work is organized as follows. In section 2, we describe the physical background. In section 3 , the numerical methods are described in detail. In section 4 we validate the field solver without particles on curved meshes with a dielectric material as well as its interaction with a single particle and with particles in a plasma sheath. In section 5, the application of the scheme is demonstrated by simulating a gridded ion thruster with a complex geometry.

\section{Theory}

Collision free electrostatic plasmas are described by the Vlasov-Poisson equation

$$
\frac{\partial f_{\alpha}}{\partial t}+\boldsymbol{v}_{\alpha} \frac{\partial f_{\alpha}}{\partial \boldsymbol{x}_{\alpha}}+\frac{\mathbf{F}}{m_{\alpha}} \frac{\partial f_{\alpha}}{\partial \boldsymbol{v}_{\alpha}}=0 .
$$

Here, $f_{\alpha}=f_{\alpha}(\boldsymbol{x}, \boldsymbol{v}, t)$ is the particle distribution function of species $\alpha$ depending on the position $\boldsymbol{x}$, velocity $\boldsymbol{v}$ and time $t$. Furthermore, $m$ is the particle mass 
and $\mathbf{F}$ is the electrostatic Lorentz force given by

$$
\mathbf{F}=q_{\alpha} \boldsymbol{E},
$$

with the particle charge $q$ and the electric field $\boldsymbol{E}$. In this work, we neglect electromagnetic waves and consider the electrostatic approximation, assuming that the electric field is irrotational [10]:

$$
\nabla \times \boldsymbol{E}=0 \quad \Rightarrow \quad \frac{\partial \boldsymbol{B}}{\partial t}=0 .
$$

A curl-free electric field can be described with the electrostatic scalar potential $\Phi$ :

$$
\boldsymbol{E}=-\nabla \Phi
$$

Finally, we solve Gauss's law to also take into account the interaction between electric fields and linear materials [10]:

$$
\nabla \cdot \boldsymbol{D}=\rho
$$

with the current density $\rho$ and the displacement field $\boldsymbol{D}=\varepsilon \boldsymbol{E}$. Here, $\varepsilon=\varepsilon_{0} \varepsilon_{r}$ is the product of the vacuum permittivity $\varepsilon_{0}$ and the relative permittivity of the material $\varepsilon_{r}$. Inserting eq. (4) in (5), the electric potential is given by

$$
\nabla \cdot \boldsymbol{D}=\nabla \cdot(\varepsilon \boldsymbol{E})=-\nabla \cdot(\varepsilon \nabla \Phi)=\rho .
$$

The conditions at a material discontinuity with different $\varepsilon_{+}$and $\varepsilon_{-}$are

$$
\Phi_{+}=\Phi_{-}, \quad \varepsilon_{+}(\nabla \Phi)_{+}=\varepsilon_{-}(\nabla \Phi)_{-} .
$$

The charge density $\rho$ is computed from the particle distribution function $f_{\alpha}$ of each species $\alpha$ as

$$
\rho(\boldsymbol{x}, t)=\sum_{\alpha} q_{\alpha} \int_{\mathbb{R}^{3}} f_{\alpha}(\boldsymbol{x}, \boldsymbol{v}, t) \mathrm{d}^{3} v .
$$

Furthermore, there exist different simplifications, e.g., fluid models for electrons. The most common electron fluid model is the Boltzmann relation (BR). In the BR model it is assumed that the electrons are isothermal, the pressure is 
described by an ideal gas and the electron drift velocity can be neglected [11, 12].

The advantage of the BR model is that it overcomes the different time scales between electrons and heavy particles due to the different inertias. For the given assumptions, the electron number density $n_{e}$ is given by

$$
n_{e}^{\mathrm{BR}}(\boldsymbol{x}, t)=n_{e, \text { ref }} \exp \left[\frac{\left|q_{e}\right|}{k_{B} T_{e}}\left(\Phi(\boldsymbol{x}, t)-\Phi_{\mathrm{ref}}\right)\right],
$$

with the electron temperature $T_{e}$, the potential $\Phi$ at the location of $n_{e}$, the electron charge $q_{e}$, the Boltzmann constant $k_{B}$ and a reference potential $\Phi_{\text {ref }}$ at the location $\boldsymbol{x}_{\text {ref }}$ with $n_{e}\left(\boldsymbol{x}_{\text {ref }}\right)=n_{e, \text { ref }}$. Thus, we split the total current density of $(8)$ into ion and electron contributions

$$
\begin{aligned}
\rho(\boldsymbol{x}, t) & =\rho_{I}(\boldsymbol{x}, t)+\rho(\Phi), \\
\rho_{I}(\boldsymbol{x}, t) & =\sum_{\alpha \neq e} q_{\alpha} \int_{\mathbb{R}^{3}} f_{\alpha}(\boldsymbol{x}, \boldsymbol{v}, t) \mathrm{d}^{3} v, \\
\rho(\Phi) & =q_{e} n_{e}^{\mathrm{BR}}(\boldsymbol{x}, t) .
\end{aligned}
$$

The definition of the reference point of $\Phi_{\text {ref }}$ and $n_{e, \text { ref }}$ is mostly based on the quasi-neutral assumption $n_{e}=n_{I}$ at a known location. Inserting (9) into 12 yields

$$
\rho(\Phi)=\rho_{\mathrm{ref}} \exp \left[\frac{\left|q_{e}\right|}{k_{B} T_{e}} \Phi(\boldsymbol{x}, t)\right], \quad \rho_{\mathrm{ref}}=q_{e} n_{e, \mathrm{ref}} \exp \left[\frac{-\left|q_{e}\right| \Phi_{\mathrm{ref}}}{k_{B} T_{e}}\right] .
$$

Note that the Poisson equation (6) becomes non-linear with the BR model:

$$
-\nabla \cdot(\varepsilon \nabla \Phi)=\rho_{I}+\rho(\Phi) .
$$

\section{Numerical Methods}

The code PICLas [13, which is used in this work, models the relation between the electric field $\boldsymbol{E}$ and the corresponding source $\rho$ numerically by the well-known PIC method [2]. Here, the particle distribution function is approximated as the linear combination of $n_{P}$ individual shape functions $\mathcal{S}$ with a weighting factor $w_{k}$ :

$$
f(\boldsymbol{x}, \boldsymbol{v}, t) \approx \sum_{k=1}^{n_{P}} w_{k} \mathcal{S}\left(\boldsymbol{x}_{k}(t)\right) \delta\left(\boldsymbol{v}-\boldsymbol{v}_{k}(t)\right) .
$$


This approximation can be taken as $n_{P}$ particles at positions $\boldsymbol{x}_{k}$ with velocities $\boldsymbol{v}_{k}$ and particle weights $w_{k}$. Different functions are possible and already tested for the shape function $\mathcal{S}$, see [14. The shape describes whether the particles are assumed to be very local as in the case of $\mathcal{S}\left(\boldsymbol{x}_{k}(t)\right)=\delta\left(\boldsymbol{x}-\boldsymbol{x}_{k}(t)\right)$ or to be more blurry.

In PICLas, the Poisson equation for the electrostatic potential 6 is solved on a fixed unstructured mesh using the high order HDGSEM, derived in section 3.2 .

\subsection{Solving the Poisson Equation with Boltzmann Relation}

If the Boltzmann relation is used for the electrons, eq. (14) becomes nonlinear and is solved iteratively with the Newton method. In each Newton iteration step $n$, we linearize the source term using a first order Taylor expansion

$$
\rho\left(\Phi^{n+1}\right)=\rho\left(\Phi^{n}\right)+\left(\rho_{\Phi}\right)^{n}\left(\Phi^{n+1}-\Phi^{n}\right), \quad\left(\rho_{\Phi}\right)^{n}=\left.\frac{d \rho}{d \Phi}\right|_{\Phi^{n}},
$$

so that 14 becomes

$$
\left(\rho_{\Phi}\right)^{n} \Phi^{n+1}+\nabla \cdot\left(\varepsilon \nabla \Phi^{n+1}\right)=r^{n}, \quad r^{n}=\rho_{I}-\rho\left(\Phi^{n}\right)+\left(\rho_{\Phi}\right)^{n} \Phi^{n}
$$

which is iterated until the residual is smaller than a defined tolerance $\epsilon_{\text {tol }}$ :

$$
\left\|\left(\rho_{\Phi}\right)^{n} \Phi^{n+1}+\nabla \cdot\left(\varepsilon \nabla \Phi^{n+1}\right)-r^{n}\right\|_{2}<\epsilon_{\mathrm{tol}}
$$

In each Newton step, we need to solve a linear equation system. However, since $\rho_{\Phi}{ }^{n}$ depends on the solution $\Phi^{n}$, the system matrix has to be rebuild for each Newton step. To save computational time, we use an approximation of the derivative of the source 10 . The exact derivative of the source reads

$$
\left(\rho_{\Phi}\right)=\frac{\alpha_{\mathrm{ref}}\left|q_{e}\right|}{k_{B} T_{e}} \exp \left[\frac{\left|q_{e}\right|}{k_{B} T_{e}} \Phi\right] .
$$

If $\left|q_{e}\right| \cdot|\Phi|<k_{B} T_{e}$, the derivative can be approximated by

$$
\left(\rho_{\Phi}\right) \approx\left(\rho_{\Phi}\right)_{\text {linear }}=\frac{\alpha_{\text {ref }}\left|q_{e}\right|}{k_{B} T_{e}}
$$


Note that the right hand side $r^{n}$ is still computed at each iteration with the exact source (10) and its derivative (19). Thus, once the Newton iteration converges, the solution fulfills (17) and becomes independent of the approximation of $\rho_{\Phi}$.

Nevertheless, we observe that the linear approximation can lead to a large number of Newton iterations. Using the exact derivative, but keeping it fixed for a given number of iterations is found to reduce the iteration number in many cases.

\subsection{The Hybridizable Discontinuous Galerkin Spectral Element Method}

In this section, we derive the field solver used in PICLas. The solver is based on the hybridizable DG method, with a spectral element representation on curved hexahedral elements, referred to as hybridizable discontinuous Galerkin

75 spectral element method (HDGSEM). For the non-linear case the following solution procedure must be evaluated within each step of Newton's method. In each spectral element, the solution is approximated as a Lagrange interpolation polynomial on tensor-product basis functions with Gauss or Gauss-Lobatto points, and the same points are used for the numerical integration of the variational form, yielding highly efficient implementations for high order curved three-dimensional elements. We will closely follow the derivation of the hybridizable local DG (LDG-H) method of Cockburn et al. [15].

As described above, we want to solve the electrostatic potential with the HDGSEM. In a first step, we rewrite eq. (17) into a first order system

$$
\begin{aligned}
\boldsymbol{E}+\nabla \Phi & =0 \\
\rho_{\Phi} \Phi-\nabla \cdot \boldsymbol{D} & =r, \quad \boldsymbol{D}=\varepsilon \boldsymbol{E}
\end{aligned}
$$

with the permittivity $\varepsilon$. The equations are solved on the computational domain $\Omega$, with Dirichlet and Neumann boundary conditions

$$
\left.\Phi\right|_{\partial \Omega_{\text {Dir. }}}=\Phi_{\text {Dir. }},\left.\quad \boldsymbol{D} \cdot \boldsymbol{n}\right|_{\partial \Omega_{\mathrm{Neu}} .}=\varepsilon(\boldsymbol{E} \cdot \boldsymbol{n})_{\mathrm{Neu} .} .
$$

First, we subdivide $\Omega$ into $n_{K}$ non-overlapping and conforming hexahedral elements, $K_{\nu}, \nu=1,2, \ldots, n_{K}$. The element index is omitted if not necessary, so that $K$ refers to any curved hexahedral element in physical space 
$\boldsymbol{x}=\left(x_{1}, x_{2}, x_{3}\right)$. Each element is mapped from a reference element $\hat{K}$ with reference coordinates $\boldsymbol{\xi}=\left(\xi^{1}, \xi^{2}, \xi^{3}\right) \in[-1,1]^{3}$ to physical space, and the element mapping is represented by a Lagrange interpolation polynomial of degree $N_{\text {geo }}$

$$
\boldsymbol{\xi} \mapsto \boldsymbol{x}: \boldsymbol{x}(\boldsymbol{\xi})=\sum_{m, n, o=0}^{N_{\text {geo }}} \hat{\boldsymbol{x}}_{m n o} \ell_{m}\left(\xi^{1}\right) \ell_{n}\left(\xi^{2}\right) \ell_{o}\left(\xi^{3}\right) .
$$

with element nodes $\hat{\boldsymbol{x}}_{m n o}$. From the element mapping, we deduce the covariant vectors, the Jacobian and contra-variant vectors

$$
\boldsymbol{a}_{i}=\frac{\partial \boldsymbol{x}}{\partial \xi^{i}}, \quad \mathcal{J}=\boldsymbol{a}_{i} \cdot\left(\boldsymbol{a}_{j} \times \boldsymbol{a}_{k}\right), \quad \boldsymbol{a}^{i}=\frac{1}{\mathcal{J}}\left(\boldsymbol{a}_{j} \times \boldsymbol{a}_{k}\right),
$$

with cyclic indices $i, j, k$. We can see that $\boldsymbol{a}_{i} \cdot \boldsymbol{a}^{j}=\delta_{i j}$ holds, and any vector quantity $\boldsymbol{q}$ is represented in the covariant or the contra-variant basis as

$$
\boldsymbol{q}=\boldsymbol{a}_{i} q^{i}=\boldsymbol{a}^{i} q_{i}, \quad q^{i}=\boldsymbol{q} \cdot \boldsymbol{a}^{i}, \quad q_{i}=\boldsymbol{q} \cdot \boldsymbol{a}_{i} .
$$

If not stated otherwise, we assume Einstein summation. We also introduce the unit outward pointing normal $\hat{\boldsymbol{n}}$ in reference space and define the outward pointing normal vector in physical space as well as the surface element as

$$
\boldsymbol{n}=\frac{1}{\hat{s}} \mathcal{J} \boldsymbol{a}^{i} \hat{n}_{i}, \quad \hat{s}=\left|\mathcal{J} \boldsymbol{a}^{i} \hat{n}_{i}\right| .
$$

To derive the variational form of the problem, we multiply (21) with the test functions for the electric field and its potential $\overline{\boldsymbol{E}}, \bar{\Phi}$ and integrate over the domain

$$
\begin{gathered}
\langle\boldsymbol{E}, \overline{\boldsymbol{E}}\rangle_{\Omega}+\langle\nabla \Phi, \overline{\boldsymbol{E}}\rangle_{\Omega}=0 \\
\left\langle\rho_{\Phi} \Phi, \bar{\Phi}\right\rangle_{\Omega}-\langle\nabla \cdot \boldsymbol{D}, \bar{\Phi}\rangle_{\Omega}=\langle r, \bar{\Phi}\rangle_{\Omega}
\end{gathered}
$$

We subdivide the integral over $\Omega$ in the element contributions, and look at one element $K$

$$
\begin{gathered}
\langle\boldsymbol{E}, \overline{\boldsymbol{E}}\rangle_{K}-\langle\Phi, \nabla \cdot \overline{\boldsymbol{E}}\rangle_{K}+\left\{\Phi^{*} \overline{\boldsymbol{E}} \cdot \boldsymbol{n}\right\}_{\partial K} \\
=0 \\
\left\langle\rho_{\Phi} \Phi, \bar{\Phi}\right\rangle_{K}+\langle\boldsymbol{D}, \nabla \bar{\Phi}\rangle_{K}-\left\{\left(\boldsymbol{D}^{*} \cdot \boldsymbol{n}\right) \bar{\Phi}\right\}_{\partial K}=\langle r, \bar{\Phi}\rangle_{K}
\end{gathered}
$$

where we introduced the yet to be defined unique numerical traces $\Phi^{*}, \boldsymbol{D}^{*}$, since we allow for discontinuous solutions at the element interfaces. We integrate the 
second equation by parts again, using only the inner surface evaluation $\boldsymbol{D}^{-} \cdot \boldsymbol{n}$, and get

$$
\begin{gathered}
\langle\boldsymbol{E}, \overline{\boldsymbol{E}}\rangle_{K}-\langle\Phi, \nabla \cdot \overline{\boldsymbol{E}}\rangle_{K}+\left\{\Phi^{*} \overline{\boldsymbol{E}} \cdot \boldsymbol{n}\right\}_{\partial K}=0 \\
-\langle\nabla \cdot \boldsymbol{D}, \bar{\Phi}\rangle_{K}-\left\{\left(\boldsymbol{D}^{*}-\boldsymbol{D}^{-}\right) \cdot \boldsymbol{n} \bar{\Phi}\right\}_{\partial K}+\left\langle\rho_{\Phi} \Phi, \bar{\Phi}\right\rangle_{K}=\langle r, \bar{\Phi}\rangle_{K}
\end{gathered}
$$

The main idea of the HDG method [15] is to reduce the equation system to a system solely depending on the solution at each element interface $e$. We introduce the additional unknown $\lambda$, unique on each element interface, together with its test function $\bar{\lambda}$, and write the extended system as

$$
\begin{gathered}
\langle\boldsymbol{E}, \overline{\boldsymbol{E}}\rangle_{K}-\langle\Phi, \nabla \cdot \overline{\boldsymbol{E}}\rangle_{K}+\sum_{e \in \partial K}\left\{\Phi^{*} \overline{\boldsymbol{E}} \cdot \boldsymbol{n}\right\}_{e}=0 \\
-\langle\nabla \cdot \boldsymbol{D}, \bar{\Phi}\rangle_{K}-\sum_{e \in \partial K}\left\{\left(\boldsymbol{D}^{*}-\boldsymbol{D}^{-}\right) \cdot \boldsymbol{n} \bar{\Phi}\right\}_{e}+\left\langle\rho_{\Phi} \Phi, \bar{\Phi}\right\rangle_{K}=\langle r, \bar{\Phi}\rangle_{K} \\
\left\{\llbracket \boldsymbol{D}^{*} \rrbracket \bar{\lambda}^{e}\right\}_{e}=0
\end{gathered}
$$

where the last equation imposes uniqueness of the numerical trace $\boldsymbol{D}^{*}$ at each interface $e$, with the jump definition for a vector $\boldsymbol{v}$ at an interface of two elements $e \cup \partial K(e), \partial K^{\prime}(e)$

$$
\llbracket \boldsymbol{v} \rrbracket=(\boldsymbol{v} \cdot \boldsymbol{n})^{K(e)}+(\boldsymbol{v} \cdot \boldsymbol{n})^{K^{\prime}(e)} .
$$

We make the local DG hybridizable (LDG-H) ansatz of [15] and set the numerical traces on the element interfaces to

$$
\Phi^{*}=\lambda, \quad \boldsymbol{D}^{*} \boldsymbol{n}=\boldsymbol{D}^{-} \boldsymbol{n}+\tau\left(\Phi^{-}-\lambda\right)
$$

with the stabilization parameter $\tau>0$.

We want to allow different materials of constant permittivity $\varepsilon$. We discretize the domain such that material interfaces match the element interfaces, so that inside each element, we know that $\left.\varepsilon\right|_{K}=\varepsilon_{K}$ is a constant. The unique flux condition for $\boldsymbol{D}^{*}$ already incorporates correctly the jump condition at the material interface (7). As the electric field and its test function are element-wise polynomials only, we can rewrite the first equation using $\left.\boldsymbol{E}\right|_{K}=\left.\frac{1}{\varepsilon_{K}} \boldsymbol{D}\right|_{K}$ and $\left.{ }_{90} \overline{\boldsymbol{D}}\right|_{K}=\left.\varepsilon_{K} \overline{\boldsymbol{E}}\right|_{K}$, which also restores the symmetry of the equation system. 
Inserting the numerical traces and the assumption of an constant permittivity per element $\varepsilon_{K}$, we get

$$
\begin{gathered}
\frac{1}{\varepsilon_{K}}\langle\boldsymbol{D}, \overline{\boldsymbol{D}}\rangle_{K}-\langle\Phi, \nabla \cdot \overline{\boldsymbol{D}}\rangle_{K}+\sum_{e \in \partial K}\{\lambda \overline{\boldsymbol{D}} \cdot \boldsymbol{n}\}_{e}=0 \\
-\langle\nabla \cdot \boldsymbol{D}, \bar{\Phi}\rangle_{K}+\left\langle\rho_{\Phi} \Phi, \bar{\Phi}\right\rangle_{K}-\sum_{e \in \partial K}\{\tau \Phi \bar{\Phi}\}_{e}+\sum_{e \in \partial K}\{\tau \lambda \bar{\Phi}\}_{e}=\langle r, \bar{\Phi}\rangle_{K} \\
\sum_{e \in K, K^{\prime}}\left(\{\boldsymbol{D} \cdot \boldsymbol{n} \bar{\lambda}\}_{e}+\{\tau \Phi \bar{\lambda}\}_{e}-\{\tau \lambda \bar{\lambda}\}_{e}\right)=\sum_{e \in \Omega_{\mathrm{Neu}}}\{\boldsymbol{D} \cdot \boldsymbol{n} \bar{\lambda}\}_{e},
\end{gathered}
$$

where we also included the Neumann boundary condition in the last equation. For Dirichlet boundaries, $\lambda$ can be specified directly, and for inner interfaces, the right hand side of the last equation of $(33)$ is set to zero.

As a final step to get the discrete operator, we transform the integrals and derivatives in $(33)$ to the reference element $\hat{K}$ and reference interface $\hat{e}$,

$$
\begin{array}{r}
\frac{1}{\varepsilon_{K}}\langle\mathcal{J} \boldsymbol{D}, \overline{\boldsymbol{D}}\rangle_{\hat{K}}-\left\langle\Phi, \frac{\partial\left(\mathcal{J} \boldsymbol{a}^{k} \cdot \overline{\boldsymbol{D}}\right)}{\partial \xi^{k}}\right\rangle_{\hat{K}}+\sum_{\hat{e} \in \partial \hat{K}}\{\lambda \overline{\boldsymbol{D}} \cdot \boldsymbol{n} \hat{s}\}_{\hat{e}}=0 \\
-\left\langle\frac{\partial\left(\mathcal{J} \boldsymbol{a}^{k} \cdot \boldsymbol{D}\right)}{\partial \xi^{k}}, \bar{\Phi}\right\rangle_{\hat{K}}+\left\langle\mathcal{J} \rho_{\Phi} \Phi, \bar{\Phi}\right\rangle_{\hat{K}}-\sum_{\hat{e} \in \partial \hat{K}}\{\tau \Phi \bar{\Phi} \hat{s}\}_{\hat{e}}+\sum_{\hat{e} \in \partial \hat{K}}\{\tau \lambda \bar{\Phi} \hat{s}\}_{\hat{e}}=\langle\mathcal{J} r, \bar{\Phi}\rangle_{\hat{K}} \\
\sum_{\hat{e} \in K, K^{\prime}}\left(\{\boldsymbol{D} \cdot \boldsymbol{n} \bar{\lambda} \hat{s}\}_{\hat{e}}+\{\tau \Phi \bar{\lambda} \hat{s}\}_{\hat{e}}-\{\tau \lambda \bar{\lambda} \hat{s}\}_{\hat{e}}\right)=\sum_{\hat{e} \in \Omega_{\mathrm{Neu}} .}\{\boldsymbol{D} \cdot \boldsymbol{n} \bar{\lambda} \hat{s}\}_{\hat{e}}
\end{array}
$$

We make a specific choice for the representation of the displacement field vector and the test function

$$
\boldsymbol{D}=\frac{\boldsymbol{a}_{j}}{\mathcal{J}} D^{j}, \quad \overline{\boldsymbol{D}}=\frac{\boldsymbol{a}_{i}}{\mathcal{J}} \bar{D}^{i}
$$

leading to

$$
\begin{array}{r}
\frac{1}{\varepsilon_{K}}\left\langle\boldsymbol{a}_{j} D^{j}, \frac{\boldsymbol{a}_{i}}{\mathcal{J}} \bar{D}^{i}\right\rangle_{\hat{K}}-\left\langle\Phi, \frac{\partial \bar{D}^{i}}{\partial \xi^{i}}\right\rangle_{\hat{K}}+\sum_{\hat{e} \in \partial \hat{K}}\left\{\lambda\left(\bar{D}^{i} \hat{n}_{i}\right)\right\}_{\hat{e}}=0, \\
-\left\langle\frac{\partial D^{j}}{\partial \xi^{j}}, \bar{\Phi}\right\rangle_{\hat{K}}+\left\langle\mathcal{J} \rho_{\Phi} \Phi, \bar{\Phi}\right\rangle_{\hat{K}}-\sum_{\hat{e} \in \partial \hat{K}}\{\tau \Phi \bar{\Phi} \hat{s}\}_{\hat{e}}+\sum_{\hat{e} \in \partial \hat{K}}\{\tau \lambda \bar{\Phi} \hat{s}\}_{\hat{e}}=\langle\mathcal{J} r, \bar{\Phi}\rangle_{\hat{K}}, \\
\sum_{\hat{e} \in K, K^{\prime}}\left(\left\{\left(D^{j} \hat{n}_{j}\right) \bar{\lambda}\right\}_{\hat{e}}+\{\tau \Phi \bar{\lambda} \hat{s}\}_{\hat{e}}-\{\tau \lambda \bar{\lambda} \hat{s}\}_{\hat{e}}\right)=\sum_{\hat{e} \in \Omega_{\mathrm{Neu}}}\{\boldsymbol{D} \cdot \boldsymbol{n} \bar{\lambda} \hat{s}\}_{\hat{e}} .
\end{array}
$$

We apply the spectral element idea, where tensor-product basis functions with ${ }_{95}$ Gaussian quadrature points $\boldsymbol{\xi}_{m n o} m, n, o=0, \ldots N$ are used to approximate 
the integrals and also define the Lagrange polynomial basis of degree $N$ for the solution and test function $\Phi, \bar{\Phi}$ and $D^{j}, \bar{D}^{i}$. For $\lambda, \bar{\lambda}$, the $2 \mathrm{D}$ tensor-product Lagrange polynomials on the element interface are used. Since the same points are used for the solution and the integration, we can largely reduce the number of operations to build the linear matrix system, and all system matrices become very sparse. For example, the mass matrix in the first equation of 36 becomes block-diagonal, with $i, j=1,2,3$ block entries $\frac{1}{\varepsilon_{K}} \omega_{m} \omega_{n} \omega_{o}\left[\frac{1}{\mathcal{J}} \boldsymbol{a}_{i} \cdot \boldsymbol{a}_{j}\right]_{\boldsymbol{\xi}_{m n o}}$.

Finally, after applying the spectral element ansatz, equation (36) can be written as a symmetric linear equation system, where we collect all degrees of freedom into a vector, consisting of the unknowns in the elements $\underline{D}, \underline{\Phi}$ and the unknowns on the element interfaces $\underline{\lambda}$

$$
\left[\begin{array}{ccc}
\underline{\underline{\mathcal{A}}} & \underline{\underline{\mathcal{B}}}^{T} & \underline{\underline{\mathcal{C}}}^{T} \\
\underline{\underline{\mathcal{B}}} & \underline{\underline{\mathcal{D}}} & \underline{\underline{\mathcal{E}}}^{T} \\
\underline{\underline{\mathcal{C}}} & \underline{\underline{\mathcal{E}}} & \underline{\underline{\mathcal{F}}}
\end{array}\right]\left[\begin{array}{c}
\underline{\mathrm{D}} \\
\underline{\Phi} \\
\underline{\lambda}
\end{array}\right]=\left[\begin{array}{c}
0 \\
\underline{r} \\
\underline{\mathrm{D}}_{N}
\end{array}\right] .
$$

We reduce the full system to a symmetric system for the unknowns on the element interfaces $\underline{\lambda}$ only,

$$
\begin{array}{r}
{\left[\underline{\underline{\mathcal{E}}} \underline{\underline{\mathcal{D}}}^{-1} \underline{\underline{\mathcal{E}}}^{T}+\underline{\underline{\mathcal{C}}} \underline{\underline{\mathcal{A}}}^{-1} \underline{\underline{\mathcal{C}}}^{T}-\underline{\underline{\mathcal{F}}}\right] \underline{\lambda}=\underline{\underline{\tilde{\mathcal{E}}}} \underline{\underline{\mathcal{D}}}^{-1} \underline{\underline{r}}+\underline{\mathrm{D}}_{N}} \\
\underline{\underline{\mathcal{\mathcal { D }}}}=\underline{\underline{\mathcal{D}}}-\underline{\underline{\mathcal{B}}} \underline{\underline{\mathcal{A}}}^{-1} \underline{\underline{\mathcal{B}}}^{T}, \quad \underline{\underline{\mathcal{E}}}=\underline{\underline{\mathcal{E}}}-\underline{\underline{\mathcal{C}}} \underline{\underline{\mathcal{A}}}^{-1} \underline{\underline{\mathcal{B}}}^{T} .
\end{array}
$$

Note that $\underline{\underline{\mathcal{A}}}$ is diagonal with $3 \times 3$ blocks and $\underline{\underline{\mathcal{B}}}, \underline{\underline{\mathcal{D}}}, \underline{\underline{\underline{\mathcal{D}}}}$ are element block-diagonal matrices and therefore $\underline{\underline{\mathcal{A}}}, \underline{\underline{\underline{\mathcal{D}}}}$ are easy to invert. The HDGSEM solver is parallelized with MPI, element matrices are build locally, and we solve the reduced $\underline{\lambda}$ system with an iterative conjugate gradient solver, using either a diagonal or block-Jacobi preconditioner.

Once the solution $\lambda$ at the element interfaces is known, the evaluation of the potential and the electric field is a post-processing step that only involves fast element-local solves of the first two equations in (33), which also benefit from the tensor-product structure and the diagonal mass matrices. 


\section{Validation results}

In this section, we first investigate the properties of the electrostatic field solver without particles, demonstrating h- and p-convergence on three-dimensional curved meshes of a dielectric sphere. Secondly, we investigate the interaction of a single point source with a planar dielectric material and the interaction of particles and electric potential including the BR assumption in a plasma sheath.

\subsection{Dielectric Sphere}
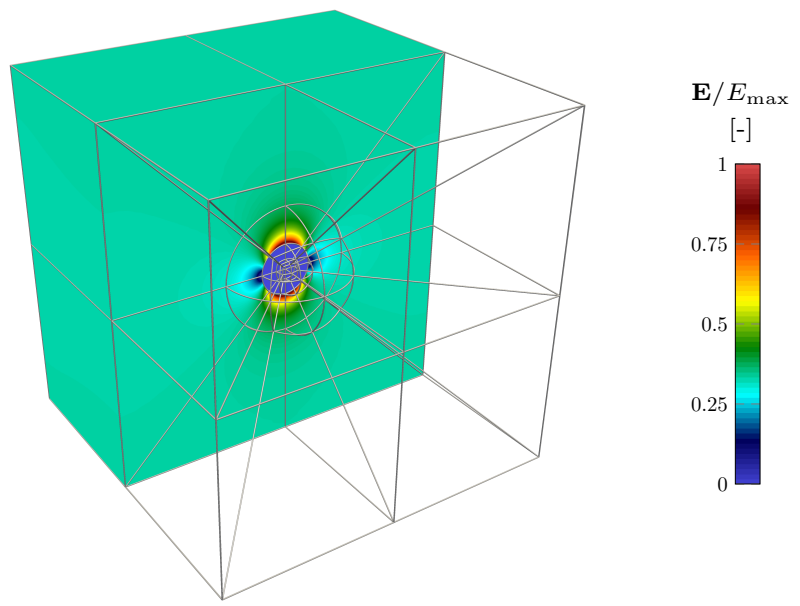

Figure 1: Cutaway of the electric field through a dielectric sphere and curvilinear mesh consisting of 80 cells with polynomial degree of the geometry $N_{\text {geo }}=2$ and solution $N=4$.

To study the HDG solver properties without particles, we validate the method against the analytical solution of a dielectric sphere with radius $R$ in a constant electric field $\mathbf{E}_{0}=\left(0,0, E_{0}\right)^{T}$, found in [10]. The electric potential reads as

$$
\Phi(x, y, z)= \begin{cases}-\left(\frac{3}{\tilde{\varepsilon}+2}\right) E_{0} z & r \leq R, \\ \left(\frac{\tilde{\varepsilon}-1}{\tilde{\varepsilon}+2} \frac{R^{3}}{r^{3}}-1\right) E_{0} z & r>R\end{cases}
$$

for the potential inside and outside of the sphere, with $r=\sqrt{x^{2}+y^{2}+z^{2}}$ and ${ }_{120} \tilde{\varepsilon}=\frac{\varepsilon_{\text {in }}}{\varepsilon_{\text {out }}}$ being the ratio of permittivities. Note that due to the material jump, 


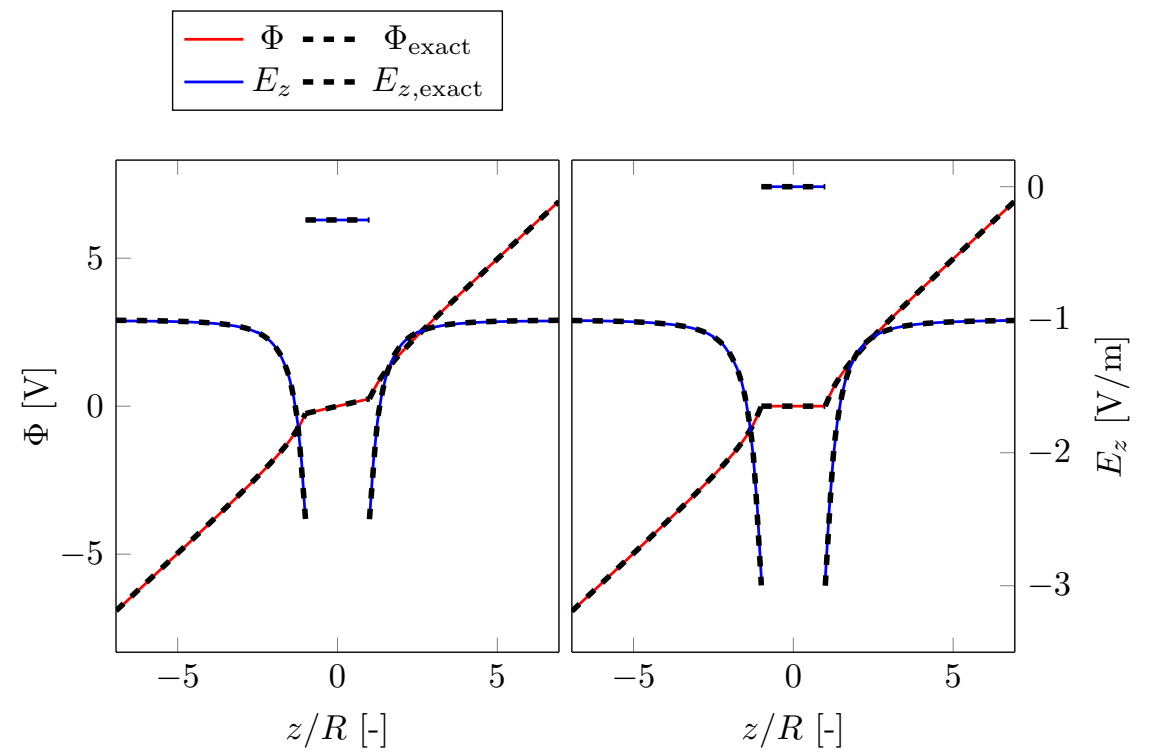

Figure 2: Comparison of numerical and analytical solution for the potential $\Phi$ and the electric field $E_{z}$ in $z$-direction for $x=y=0, \tilde{\varepsilon}=10$ (left) and $\tilde{\varepsilon}=100000$ (right) for an externally applied field of $E_{0}=-1 \mathrm{~V} / \mathrm{m}$.

the potential field exhibits a kink that results in a discontinuous curve progression of the electric field. The block-structured curved meshes were generated with the open source high-order preprocessor HOPR [16], where polynomial degree of the element mapping $N_{\text {geo }}$ is an input parameter and the interpolation nodes are placed exactly on the spherically shaped material interface.

Figure 1 depicts the mesh and the potential field solution for $\tilde{\varepsilon}=10$ and $E_{0}=-1 \mathrm{~V} / \mathrm{m}$ with $N_{\text {geo }}=2$ and polynomial degree of the solution $N=$ 4. Figure 2 compares the analytical with the numerical solution along the $z$-axis showing excellent agreement for two different values of $\tilde{\varepsilon}$. The value ${ }_{130} \tilde{\varepsilon}=100000$ demonstrates that the proposed solver is able to cover the whole range of typically employed dielectric materials.

For the convergence study, we always compute the $L_{2}$ error norm of the potential field, and we use the meshes depicted in Figure 3, where a slice of the coarsest mesh and two refinement levels are shown. The coarsest mesh consists 

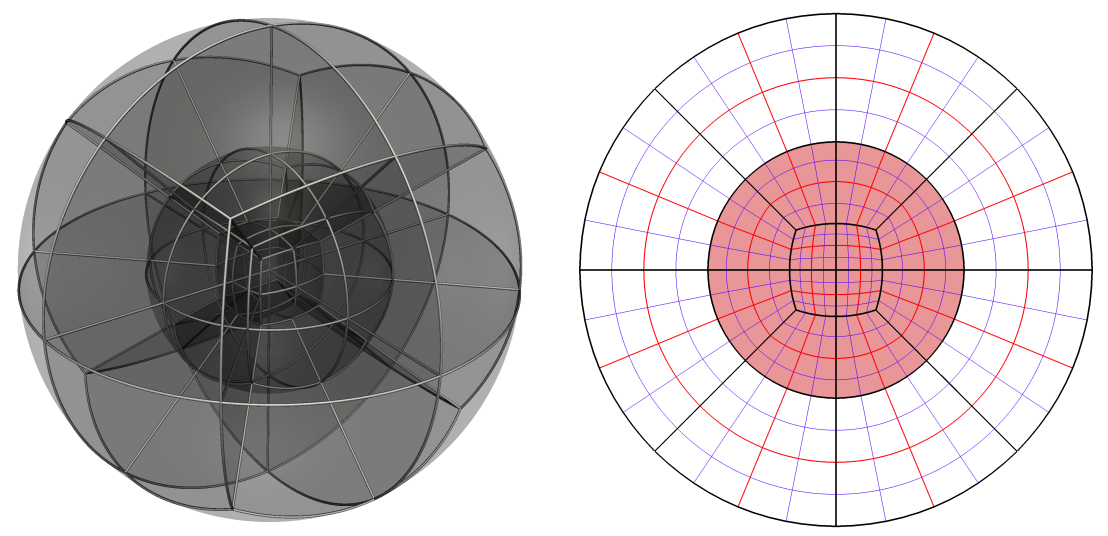

Figure 3: Fully spherical geometry. Three-dimensional view (left) of the coarse mesh with $N_{\text {geo }}=5$ and slice (right) showing three different mesh resolutions, coarse (black), medium (red) and fine (blue) for $N_{\text {geo }}=2$. The shaded area shows the dielectric region.

of 56 grid cells and each refinement step increases the number of cells by a factor of 8 . Note that in each refinement step, the interpolation points at the material interface are positioned exactly on the sphere surface, thus the geometry error converges with the order $\mathcal{O}\left(N_{\text {geo }}+1\right)$.

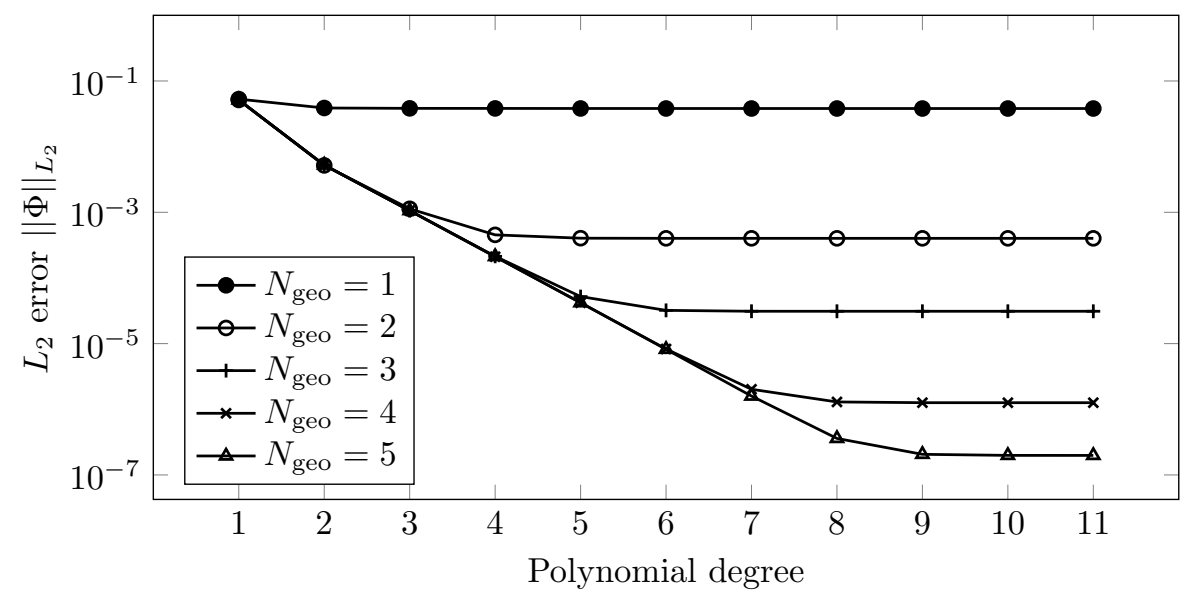

Figure 4: p-convergence of the $L_{2}$ error for the potential $\Phi$ for the dielectric sphere with $\tilde{\varepsilon}=10$, for $N_{\text {geo }}=1, \ldots, 5$.

As shown in Figure 4, we investigate first the p-convergence on the coarsest 


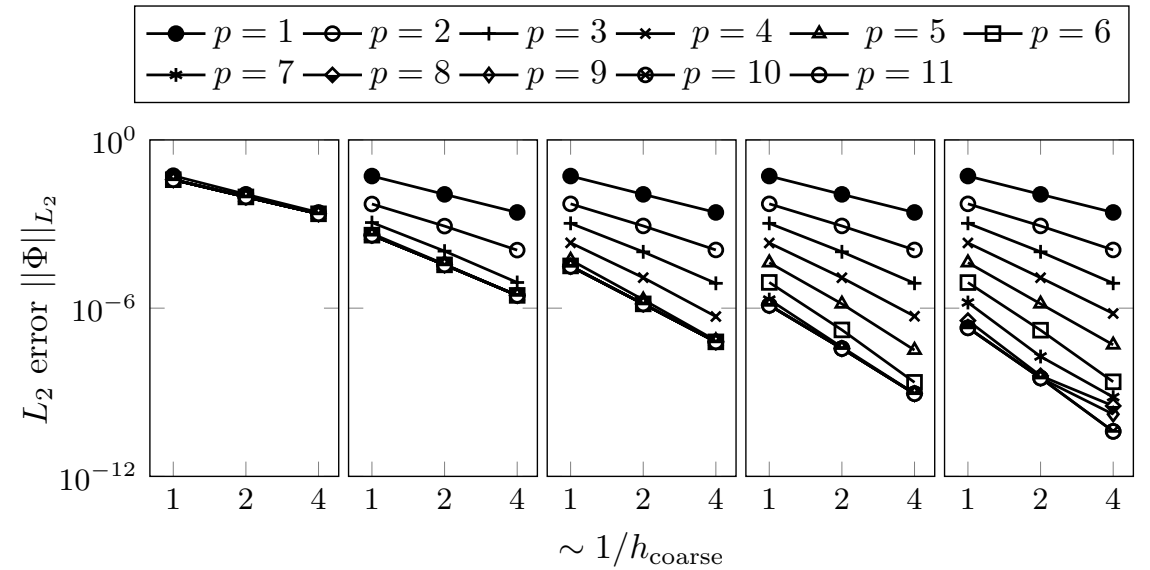

Figure 5: h-convergence of the $L_{2}$ error of the potential $\Phi$ for the dielectric sphere with $\tilde{\varepsilon}=10$, for $N_{\text {geo }}=1, \ldots, 5$ (left to right).

mesh, where the polynomial degree of the solution is increased, for different geometry approximations $N_{\text {geo }}=1, \ldots, 5$. It clearly shows that high order solutions are more accurate, but only if the curved geometry is also represented by high order polynomials of at least $N_{\text {geo }}=N$. The error is dominated by the geometrical approximation error, and an accuracy improvement is found up to $N=2 N_{\text {geo }}$. This is the limit where metric terms can be exactly interpolated by the solution points [17].

Figure 5 shows $L_{2}$ error norms for the potential field for the three different meshes ( $h$-convergence) for different geometry and solution approximation $(1 \leq$ $N_{\text {geo }} \leq 5$ and $1 \leq N \leq 11$ ). For all meshes, a higher degree $N$ improves the solution. However, the order of convergence for $N \geq N_{\text {geo }}$ is always dominated by the geometry $\mathcal{O}\left(N_{\text {geo }}+1\right)$, as shown in Table 1 , where all convergence rates are summarized.

Finally, the runtimes for matrix initialization and solve of the reduced $\lambda$ system (38) are reported in Table 2 , for four selected simulations of the dielectric sphere. We use a CG solver with a diagonal-Jacobi preconditioner, and the abort criterion was set to machine precision. The simulations were run with a single MPI rank on one Cray XC40 node and also on 2 nodes and 48 MPI ranks. We compare a high order solution on the coarse mesh with a lower order solution 
Table 1: Experimental order of convergence $\mathcal{O}$ for different polynomial degrees $N$ and geometrical degrees $N_{\text {geo }}$ for the case of a dielectric sphere.

\begin{tabular}{c|ccccccccccc}
\hline$N_{\mathrm{g} \odot \mathrm{N}}$ & 1 & 2 & 3 & 4 & 5 & 6 & 7 & 8 & 9 & 10 & 11 \\
\hline 1 & 2.18 & 2.03 & 2.02 & 2.02 & 2.02 & 2.02 & 2.02 & 2.02 & 2.02 & 2.02 & 2.02 \\
2 & & 2.73 & 3.55 & 3.66 & 3.57 & 3.57 & 3.57 & 3.57 & 3.57 & 3.57 & 3.57 \\
3 & & & 3.55 & 4.37 & 4.77 & 4.5 & 4.48 & 4.48 & 4.47 & 4.47 & 4.47 \\
4 & & & & 4.34 & 5.18 & 5.92 & 5.56 & 5.25 & 5.23 & 5.23 & 5.23 \\
5 & & & & & 4.87 & 5.88 & 5.6 & 5.05 & 5.14 & 6.13 & 6.13 \\
\hline
\end{tabular}

Table 2: Runtimes of high order solution on a coarse mesh (56 cells, 156 sides) and low order solution on a fine mesh (3584 cells, 10560 sides) with similar $L_{2}$ errors, for the dielectric sphere case. Runtime on 1 Cray XC40 node with 1 MPI rank and in brackets on two nodes with 48 MPI ranks. Note: $\operatorname{DOF}(\Phi)=(N+1)^{3} \#$ cells, $\operatorname{DOF}(\lambda)=(N+1)^{2} \#$ sides.

\begin{tabular}{cccc|c|l|l|r}
\hline$N\left(N_{\text {geo }}\right)$ & $\#$ cells & $\operatorname{DOF}(\Phi)$ & $\operatorname{DOF}(\lambda)$ & $L_{2}(\Phi)$ & Init $[\mathrm{s}]$ & Solve $[\mathrm{s}]$ & $\#$ iter. \\
\hline $5(5)$ & 56 & $12.1 \cdot 10^{3}$ & $5.62 \cdot 10^{3}$ & $4.03 \cdot 10^{-5}$ & 0.24 & 0.66 & 576 \\
$2(2)$ & 3584 & $96.8 \cdot 10^{3}$ & $95.0 \cdot 10^{3}$ & $11.2 \cdot 10^{-5}$ & 0.46 & 35.7 & 1113 \\
\hline $9(5)$ & 56 & $56.0 \cdot 10^{3}$ & $15.6 \cdot 10^{3}$ & $5.54 \cdot 10^{-8}$ & $7.22\{0.38\}$ & $13.4\{0.69\}$ & 756 \\
$5(5)$ & 3584 & $774 \cdot 10^{3}$ & $380 \cdot 10^{3}$ & $44.4 \cdot 10^{-8}$ & $14.9\{0.37\}$ & $310\{12.1\}$ & 2029 \\
\hline
\end{tabular}


of similar $L_{2}$ error on a finer mesh. For $N=5$, a similar solution is found with $N=2$ on a finer mesh with 64 times more cells. Secondly, at higher resolution, we compare a solution with $N=9$ on the coarse mesh to $N=5$ on the fine mesh. The matrix initialization times are very similar, meaning that the increased cost per high order grid cell balances with the lower number of cells. We want to point out that the solver needs less iterations and less time per iteration for the high order simulation compared to the low order simulation, still producing more accurate results. The parallel simulation times show the same behavior, despite a large load imbalance for the coarse mesh, where only 56 cells and 156 sides are distributed on 48 MPI ranks.

\subsection{Point Source in Dielectric Medium}

In order to validate the Poisson solver with source terms, we consider a test case with two dielectric regions $\varepsilon_{1}$ for $z>0$ and $\varepsilon_{2}$ for $z<0$ with a single point charge $q$ at position $(0,0, d)$. The analytical solution for this problem is found in [10] and reads

$$
\Phi= \begin{cases}\frac{q}{4 \pi \varepsilon_{1}}\left(\frac{1}{\sqrt{r^{2}+(d-z)^{2}}}-\left(\frac{\varepsilon_{2}-\varepsilon_{1}}{\varepsilon_{1}+\varepsilon_{2}}\right) \frac{1}{\sqrt{r^{2}+(d+z)^{2}}}\right) & , z \geq 0 \\ \frac{q}{4 \pi \varepsilon_{2}}\left(\frac{2 \varepsilon_{2}}{\varepsilon_{1}+\varepsilon_{2}}\right) \frac{1}{\sqrt{r^{2}+(d-z)^{2}}} & , z<0\end{cases}
$$

with $r=\sqrt{x^{2}+y^{2}}$.

The three-dimensional setup consists of a domain of size $\Delta x \times \Delta y \times \Delta z=$ $12 \mathrm{~m} \times 12 \mathrm{~m} \times 24 \mathrm{~m}$ which is discretized by $3 \times 3 \times 6$ grid cells with a polynomial degree $N=9$. Figure 6 illustrates the domain and the potential field solution resulting from a positively charged particle placed in a dielectric region with $\varepsilon_{1}=$ $200 \mathrm{C} / \mathrm{Vm}$ whereas the second region is defined with $\varepsilon_{2}=1 \mathrm{C} / \mathrm{Vm}$. Figure 7 shows the particle positioned in the dielectric region, the grid cells and a line plot along the $z$-axis comparing the analytical with the numerical solution. The singularity in the numerical solution is smoothed by the use of a polynomial 

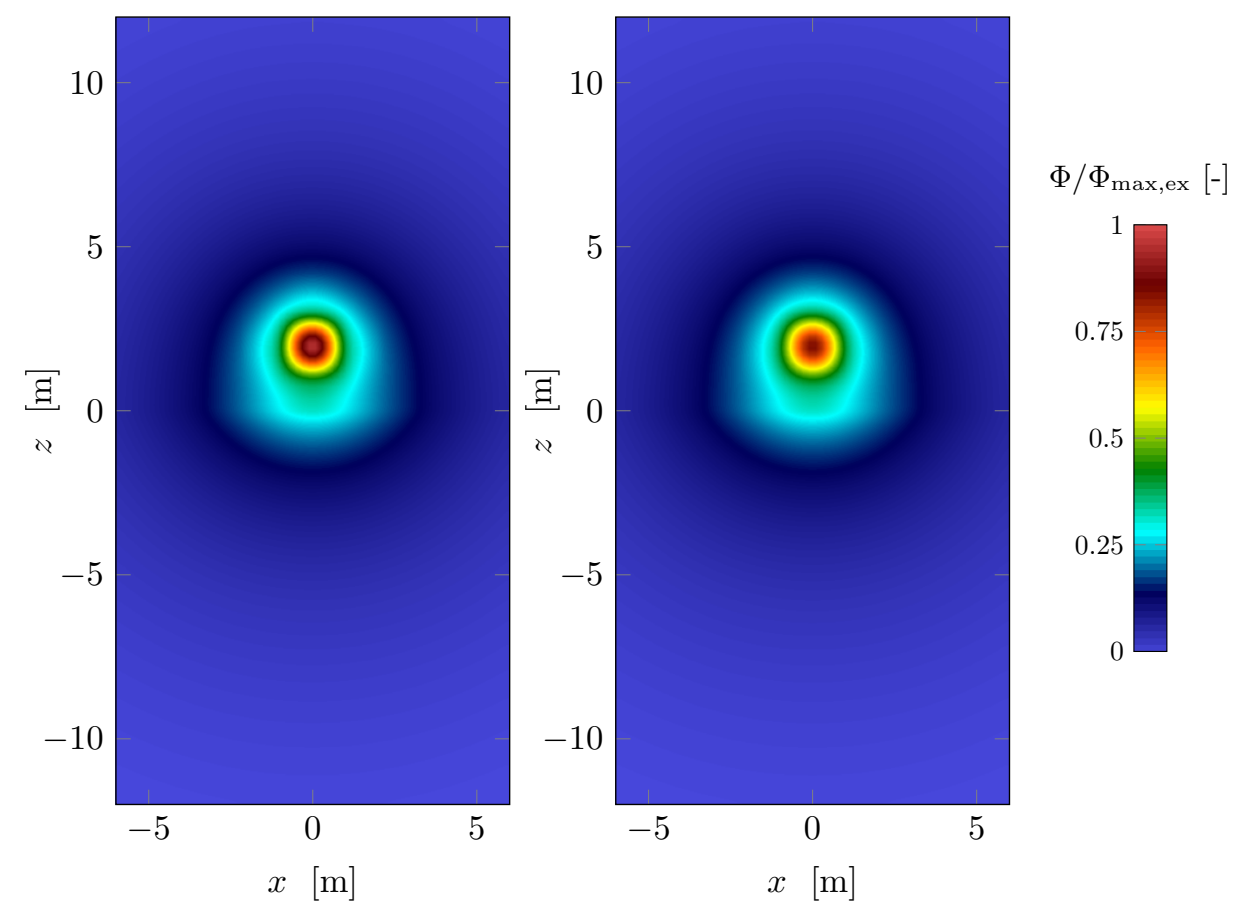

Figure 6: Single charged particle in dielectric region with $q=1 \mathrm{C}, d=2 \mathrm{~m}, \varepsilon_{1}=200 \mathrm{C} / \mathrm{Vm}$ and $\varepsilon_{2}=1 \mathrm{C} / \mathrm{Vm}$. Slice in the $x$-y-plane showing the analytical (left) and numerical (right) numerical solution. The scaling factor $\Phi_{\max , \mathrm{ex}}=1 \cdot 10^{-3} \mathrm{~V}$ is the maximum value of the analytical solution on the interpolation points. 
shape function [14], specifically the form found in [18]

$$
S(r)=\frac{1}{2 \beta_{0}(1.5, \alpha+1) \pi r_{S F}^{3}}\left[1-\left(\frac{r}{r_{S F}}\right)^{2}\right]^{\alpha},
$$

where $\beta_{0}(x, y)$ denotes the beta function, $r_{\mathrm{SF}}$ the cut-off radius and $r$ the distance between a particle's position and a DOF to which the charge density is assigned. In this setup, a cut-off radius of $r_{\mathrm{SF}}=0.8 \mathrm{~m}$ and the exponent $\alpha=3$ are chosen. This means that the shape function spans only over one cell with the largest deviation from the analytical solution due to the singularity whereas outside the cell excellent agreement is found between the simulation and the analytical solution.
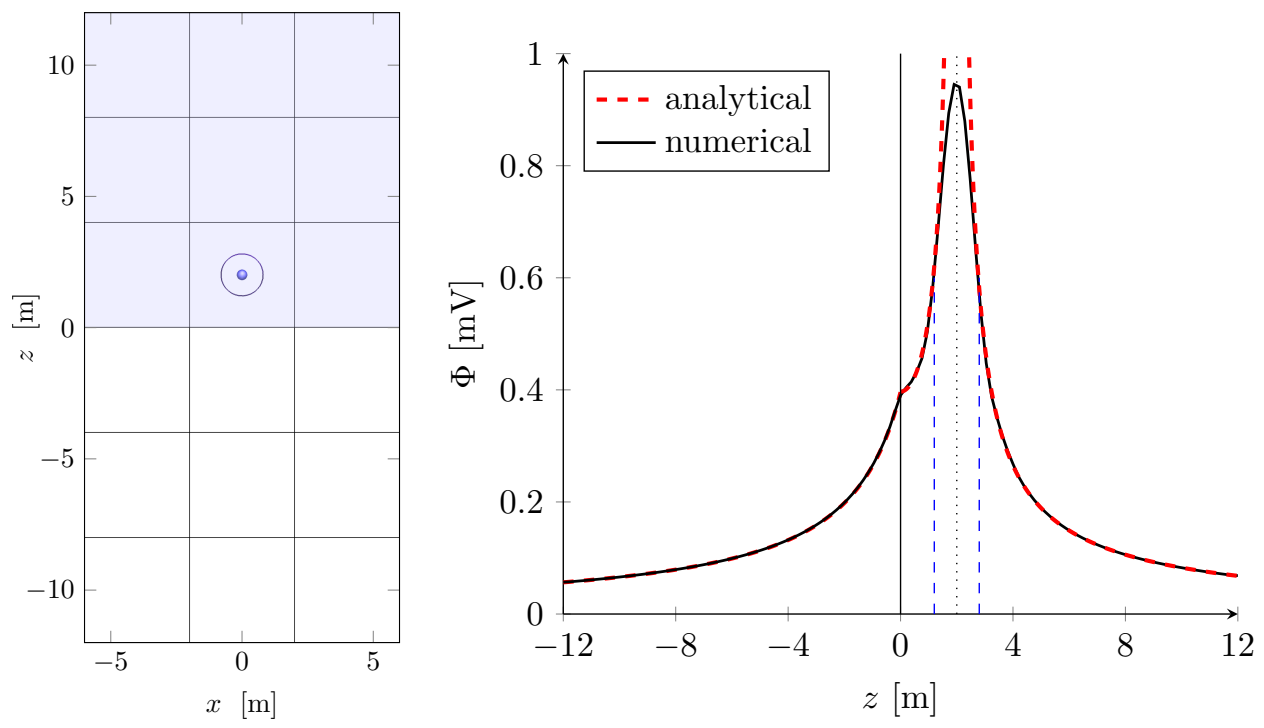

Figure 7: Single charged particle in dielectric region (left) with a shape function radius, indicated as a circle, of $r_{\mathrm{SF}}=0.8 \mathrm{~m}(\approx 34 \mathrm{DOF}$ within the shape function sphere for $N=9)$ where the shaded area indicates the dielectric region. Line plot along the $z$-axis (right) comparing the analytical with the numerical solution for $q=1 \mathrm{C}, d=2 \mathrm{~m}, \varepsilon_{1}=200 \mathrm{C} / \mathrm{Vm}$ and $\varepsilon_{2}=1 \mathrm{C} / \mathrm{Vm}$. 


\subsection{Plasma Sheath}

The test case of a plasma sheath allows us to validate the full solver, where fields and particles are coupled. Furthermore, the BR model for electrons is used, so also the nonlinear field solve is tested.

The plasma sheath describes the shielding of a perfectly conducting wall in an unmagnetized and collisionless plasma consisting of electrons and singly charged ions [19, 20]. Due to a negative potential on a perfectly conducting wall, the electrons are repelled by the wall whereas the positive ions are accelerated towards the wall. This charge separation forms a potential that can be described analytically for a 1D case using the Boltzmann relation by [19]:

$$
\frac{1}{2} \chi^{\prime 2}=\vartheta^{2}\left[\left(1+\frac{2 \chi}{\vartheta^{2}}\right)^{1 / 2}-1\right]+\mathrm{e}^{-\chi}-1 .
$$

Here, $\chi=-q_{e} \Phi /\left(k_{B} T_{e}\right), \chi^{\prime}=-q_{e} \partial_{x} \Phi /\left(k_{B} T_{e}\right)$ and $\vartheta=v_{I} / \sqrt{k_{B} T_{e} / m_{I}}$ with ions entering the sheath with the velocity $v_{I}$ and mass $m_{I}$. The simulation is done using a pseudo 1D mesh with one cell in $y$ and $z$-direction as well as periodic boundary conditions and 20 cells in $\mathrm{x}$ direction. The length of the mesh is $L=0.03 \mathrm{~m}$ with the Dirichlet boundary conditions for the potential of $\Phi(x=0)=0 \mathrm{~V}$ and $\Phi(x=L)=-0.18011 \mathrm{~V}$. The inflow boundary conditions at $x=0$ of the ions and the electrons are described in Table 3 . At $x=L$ an open particles boundary condition is used. For the BR model, the reference

Table 3: Plasma sheath inflow boundary conditions.

\begin{tabular}{lll}
\hline & Electrons & Ions \\
\hline $\mathrm{n}_{\infty}\left[\mathrm{m}^{-3}\right]$ & $1.0 \cdot 10^{12}$ & $1.0 \cdot 10^{12}$ \\
$\mathrm{~T}[\mathrm{~K}]$ & 1000 & 1000 \\
$\mathrm{~m}[\mathrm{~kg}]$ & $9.109 \cdot 10^{-31}$ & $1.673 \cdot 10^{-27}$ \\
$\mathrm{v}[\mathrm{m} / \mathrm{s}]$ & 0 & 11492.19 \\
\hline
\end{tabular}

values are defined at the inflow $x=0$ as $n_{e, r e f}=1.0 \cdot 10^{12} \mathrm{~m}^{-3}$ and $\Phi_{\text {ref }}=0 \mathrm{~V}$ with isothermal electron temperature $T_{e}=1000 \mathrm{~K}$. The simulations are done 
with a particle weighting $\omega_{k}=10$ resulting in $\approx 2.67 \cdot 10^{4}$ ions in the steady state that is reached after $\approx 7.5 \cdot 10^{-6} \mathrm{~s}$ simulation time. The used time step is $\Delta t=1 \cdot 10^{-7} \mathrm{~s}$ and the polynomial degree $N=5$. The results of the simulation as well as the analytical solution are shown in Figure 8 and show excellent agreement.

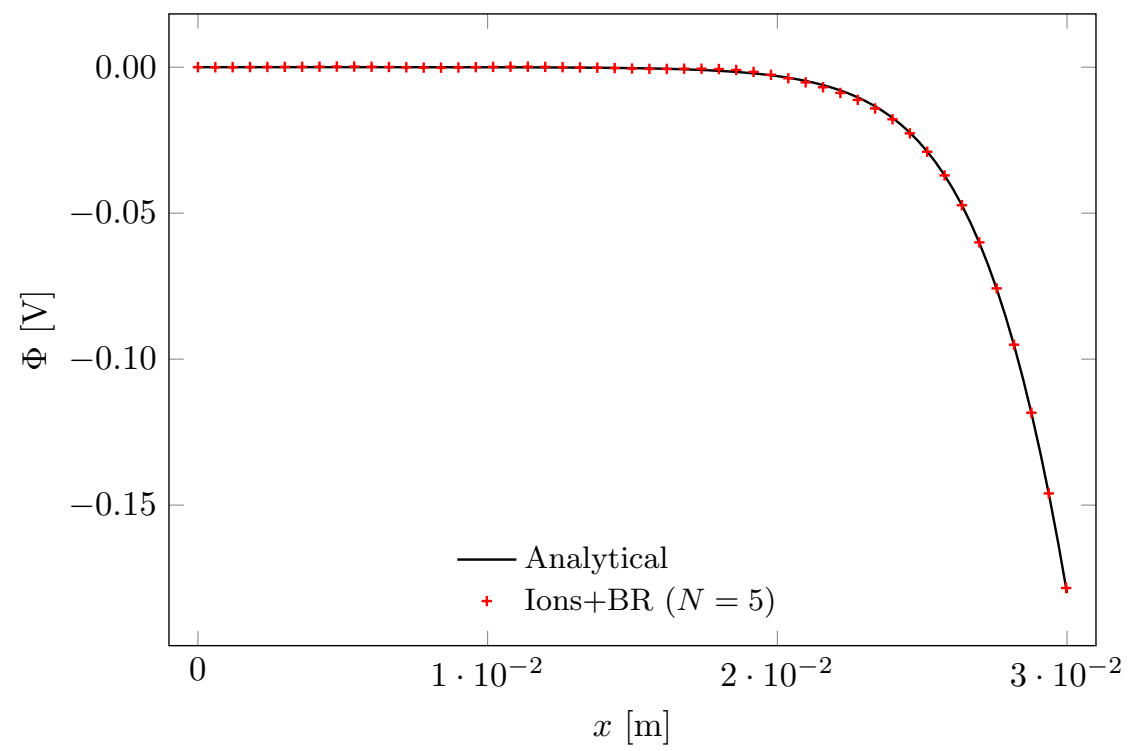

Figure 8: Plasma sheath result of the simulation using the BR model compared with the analytical solution.

\section{Ion Optics}

The simulation of ion optics, as used in gridded ion thrusters, is well-suited to demonstrate the capabilities of the HDG-based PIC solver, since it allows to analyze the interaction between both field solver and particle routines when applied to a complex three-dimensional geometry. The apertures have a cylindrical shape, thus a curved body-fitted mesh to represent the wall boundary is needed for high order accuracy of the field solver, as shown in section 4.

The quality of the simulation results depends on all PIC-specific aspects; the interpolation of charge densities from particles onto the mesh ("deposition"), 
the accurate calculation of the electric field, and finally the field evaluation and temporal integration of the equations of motion to update the particle positions. Each of these parts is sensitive to the spatial discretization. $4^{t h}$-order shape functions are used for deposition as already described in sec. 4.2 , which need to be interpolated by a sufficient number of high order solution points [14]. In this section, we investigate the convergence behavior of the charge density field for varied polynomial degree including the influence of the statistical uncertainty due the particle approach.

\subsection{Full Simulation}

215

In 1, the HDG-PIC method was already applied to simulate a full ion thruster optic, based on a RIT $\mu \mathrm{X}$ EBB from ArianeGroup GmbH. Figure 9 depicts the simulated extracted ion beams (iso-surfaces of number densities) within the half geometry of the thruster. The discharge chamber has a diameter of $40 \mathrm{~mm}$ and is closed by a two-grid system with 37 apertures in a pattern within ${ }_{220}$ a diameter of approximately $20 \mathrm{~mm}$. This full simulation included $\approx 4.3 \cdot 10^{6}$ simulation particles for the ions as well as 90758 mesh cells with a polynomial degree of $N=4$ resulting in $\approx 1.1 \cdot 10^{7}$ high-order interpolation points and illustrates the applicability to large problem sizes. The BR and fully kinetic simulations were compared and resulted in good aggrement between each other.

${ }_{225}$ The computation with $\mathrm{BR}$ took $t_{C P U} \approx 1$ hour to reach steady state on 2400 cores of a Cray XC40 and allowed to used a 100 times greater time step $\Delta t$ compared to the fully kinetic simulation. Independent from the electron model, the ratio $t_{C P U} / \Delta t$ was constant and, therefore, the BR saved the factor of 100 also in computational cost.

\subsection{Simplified Simulation Setup}

For the following analysis, we want to keep the geometrical complexity while the domain size is reduced by assuming two different simplified domains, both representing an effectively infinite pattern of grid apertures taken from the full simulation setup. First, the computational domain ('A' in Fig. 10 spans over 
is assumed at the 'inflow A' plane. The second domain ('B' in Fig. 10) is limited to an extent from which the low potentials would repel all electrons. Therefore, electrons can be completely excluded from the simulations and only ions are considered. The upstream 'inflow B' plane between first and second full simulation. The boundary conditions for the field solver are equipotential at the inflow and zero normal gradient at the outflow. Additional zero gradient conditions in $\mathrm{x}-\mathrm{y}$ and $\mathrm{x}-\mathrm{z}$ planes reduce the topology to two quarter apertures.

Figure 11 depicts the surface mesh where boundary conditions (BCs) are applied, with corresponding definitions in Table 12 . For particles, open BCs remove crossing particles from the simulation, whereas symmetry conditions perform specular reflection. For domain 'A', a mesh with 2416 cells (22 cells along the x-axis) was considered, domain 'B' consists of 928 cells (10 cells along the x-axis), both with $N_{\text {geo }}=2$. The temporal integration of particle trajecto$4^{\text {th }}$ order with a time step of $\Delta t=5 \cdot 10^{-9} \mathrm{~s}$, estimated from the ion velocity and electric field gradients. In the inflow region of domain 'A', the upstream quasi-neutral state has an assumed bulk velocity of $2500 \mathrm{~m} / \mathrm{s}$, ion temperature of $450 \mathrm{~K}$, and electron temperature of $3.5 \mathrm{eV}$. For domain 'B', truncated ion profile was imposed including a mean velocity of $34700 \mathrm{~m} / \mathrm{s}$. The inflow flux was set to match a beam current of $5 \mathrm{~mA}$ for the full thruster with 37 apertures.

\subsection{Influence of field resolution on particle simulations}

Similarly as for the verfication studies in the previous chapter, the simulation results are compared between different polynomial degress $N$. The three-dimesional charge density field was chosen as characteristic, because it is sensitive to the whole interaction between field solver and particle routines, independently of the actual effect of space charges onto the electric field. Due to the Monte-Carlo-based insertion scheme of simulation particles, their distribu- 


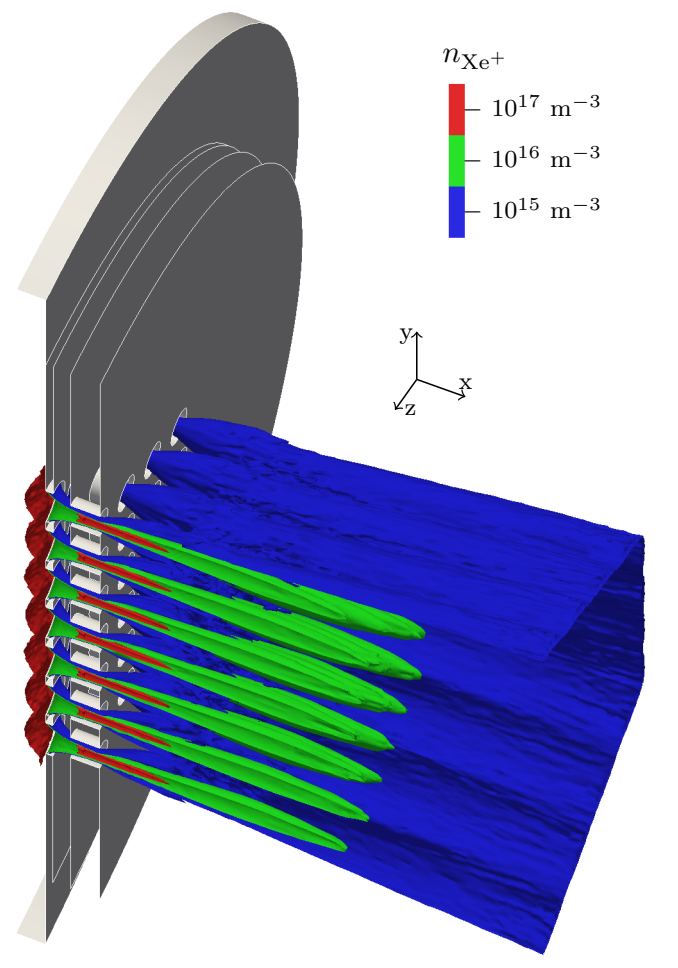

Figure 9: Iso-surfaces for number density of extracted ion beams from quasi-neutral chamber condition in a RIT- $\mu \mathrm{X}$ (full simulation [1]).

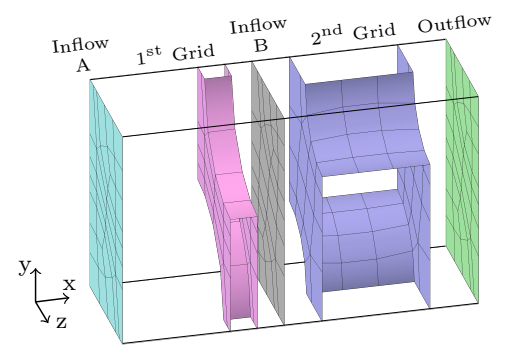

Figure 11: Surface mesh.
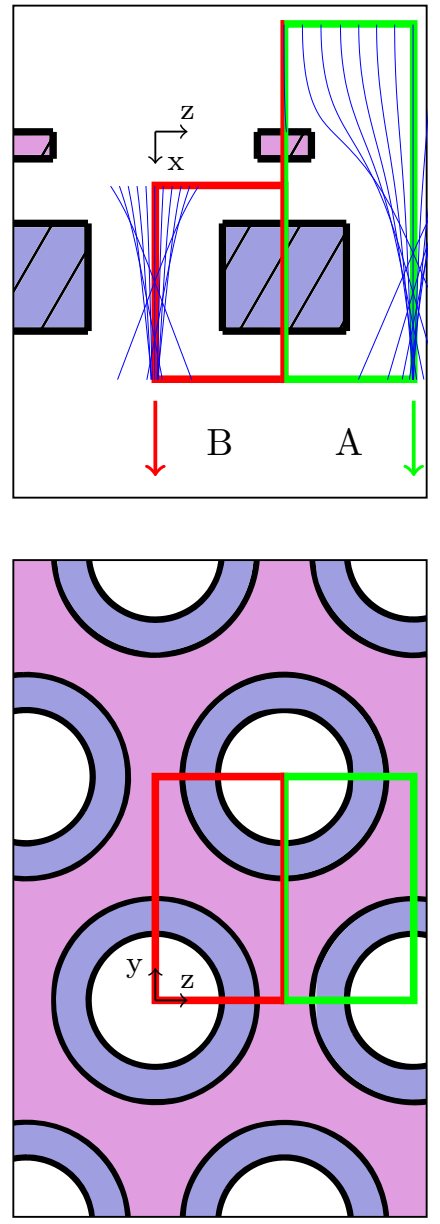

Figure 10: Simplified computational domains (red and green), above with ion trajectories (blue).

\begin{tabular}{lll}
\hline Name & Field-BC & Particle-BC \\
\hline Inflow A & $\Phi=1315 \mathrm{~V}$ & open, $\mathrm{Xe}^{+}$in \\
$1^{\text {st }}$ Grid & $\Phi=1300 \mathrm{~V}$ & open \\
Inflow B & $\Phi=500 \mathrm{~V}$ & open, $\mathrm{Xe}^{+}$in \\
$2^{\text {nd }}$ Grid & $\Phi=-300 \mathrm{~V}$ & open \\
Outflow & $\frac{\partial \Phi}{\partial \mathbf{n}}=0$ & open \\
Symmetry & $\frac{\partial \Phi}{\partial \mathbf{n}}=0$ & reflective \\
\hline
\end{tabular}


tion is affected by a statistical noise. The deposition onto the mesh respresents a smoothing approach with a certain sample size of particles with a macro particle factor of $w_{k}=500$. After having reached steady state, the sample size of the deposited charge density $\rho$ can be further increased by (arithmetically) averaging over $n_{s}$ time steps between $t_{1}$ and $t_{n_{s}}=t_{1}+n_{s} \cdot \Delta t$ at each interpolation point $\boldsymbol{x}_{j}$ of the field solver:

$$
\bar{\rho}\left(\boldsymbol{x}_{j}\right)=\sum_{i=1}^{n_{s}} \rho\left(\boldsymbol{x}_{j}, t_{i}\right) / n_{s}
$$

This averaged charge density $\bar{\rho}$ is used as comparative field based on which the deviations from a respective reference solution are evaluated. The deviation is calculated as volumetric $L_{2}$ norm $\left\|\bar{\rho}-\bar{\rho}_{\text {ref }}\right\|_{L_{2}}$, but since no analytical solution is available a solution with a very high polynomial degree $N_{\text {ref }}$ is used as reference $\bar{\rho}_{\text {ref }}$. For the comparison and integration of the $L_{2}$ norm, all solutions were interpolated onto an mesh with equidistant interpolation points of degree $N_{r e f}$. $265\left(N_{\text {ref }}+1\right)$.

Non-linear $H D G$. First, the self-consistent ion extraction from a quasi-neutral state is modeled by ions and BR electrons inside the computational domain 'A'. Figure 13 depicts the resulting contours of electric potential and ion charge density inside a symmetry plane through one grid aperture. In the inflow region with 270 $\rho_{\mathrm{Xe}^{+}}=1.3826 \cdot 10^{-2} \mathrm{C} / \mathrm{m}^{3}$, quasi-neutrality is reached since the Boltzmann relation (BR) is set to the same negative value for electrons at $\Phi_{i n}=1315 \mathrm{~V}$. About 350000 particles are simulated in steady state, mostly within the dense upstream region. Each time step, 3 Newton iteration were required for convergence of the non-linear solver using exact source derivatives.

The convergence behavior is shown in Fig. 14 for three different sample sizes ( $n_{s}=5,250$, and 3400 time steps). It can be seen that the $L_{2}$ norm levels out after having reached a certain polynomial degree, thus, even an arbitrarily higher $N$ would not result in a smaller deviation from the reference solution with $N_{\text {ref }}$. This must not be interpreted as a $N$-converged solution but rather as an integration of the statistical noise. However, it demonstrates that in the PIC 

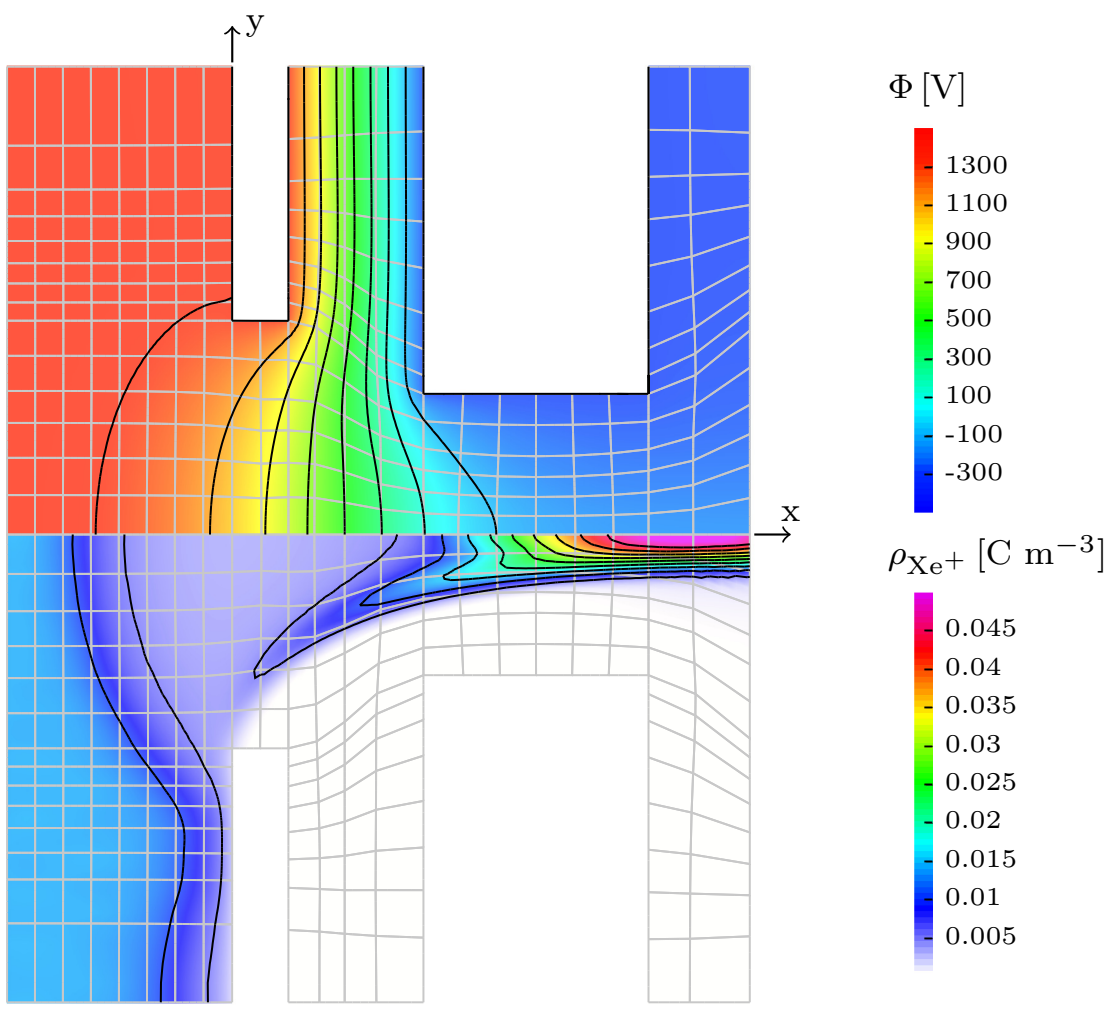

Figure 13: Contours of electric potential (above) and charge density (below) for BR case. 
application, there is a maximum reasonable $N$ beyond which the error due to the limited sample size exceeds the error based on the field resolution. Even with exactly the same numerical parameters, two solution can not match beyond their uncertainty from different random numbers due the different particle positions. To quantify this effect, the $L_{2}$ norms were not only calculated for the deviations of mean $\bar{\rho}\left(\boldsymbol{x}_{j}\right)$, but also for the corresponding 2- $\sigma$ standard error of the mean (SEM) by Eq. 44 and can be interpreted as $\left\|\bar{\rho}-\bar{\rho}_{\text {ref }}\right\|_{L_{2}}$ with a reference solution that equals $\bar{\rho}$ but is shifted exactly by ${ }^{2 \sigma} \operatorname{SEM}\left(\boldsymbol{x}_{j}\right)$ at every $\boldsymbol{x}_{j}$. Please note that for domain 'A', the $\left\|{ }^{2 \sigma} \mathrm{SEM}\right\|_{L_{2}}$ integration was only performed for mesh cells downstream of the the quasi-neutral inflow state $(x>0$ in Fig. 10) since for $x<0$ the statistical uncertainty is greater than in the evaluated region due to the smaller ion velocities and would need much larger sample sizes.

$$
\begin{aligned}
\left\|^{2 \sigma} \mathrm{SEM}\right\|_{L_{2}} & =\sqrt{\frac{\sum_{j=1}^{n_{K}} \int_{V_{j}}\left({ }^{2 \sigma} \operatorname{SEM}\left(\boldsymbol{x}_{j}\right)\right)^{2} d V_{j}}{V_{\text {total }}}}, \\
\left({ }^{2 \sigma} \operatorname{SEM}\left(\boldsymbol{x}_{j}\right)\right)^{2} & =\frac{2}{n_{s}\left(n_{s}-1\right)} \sum_{i=1}^{n_{s}}\left(\rho_{i}\left(\boldsymbol{x}_{j}\right)-\bar{\rho}\left(\boldsymbol{x}_{j}\right)\right)^{2} .
\end{aligned}
$$

Those additional $L_{2}$ norms are included in Fig. 14 as dashed lines for all $N$ and feature their proportionality to $n_{s}^{-0.5}$ and independence on $N$. It can be seen that they are very similar to the respective convergence limits and with increasing sample size the convergence limits decreases accordingly. After a $\left\|\bar{\rho}-\bar{\rho}_{\text {ref }}\right\|_{L_{2}}$ curve reaches the threshold, it simply fluctuates within the same order of magnitude as of the limit itself.

Linear HDG. Eventually, the truncated domain 'B' is considered which enables to neglect electrons inside the domain. After having reached steady-state with a simulation particle number of about 35000, charge densities were averaged with $n_{s}=250$ as described before. Again, Figure 15 depicts the $L_{2}$ norms for different $N$ with the highest simulated $N$ as reference. The diagram shows a very similar convergence behavior as the one with $\mathrm{BR}$ electrons inside domain 'A'. 


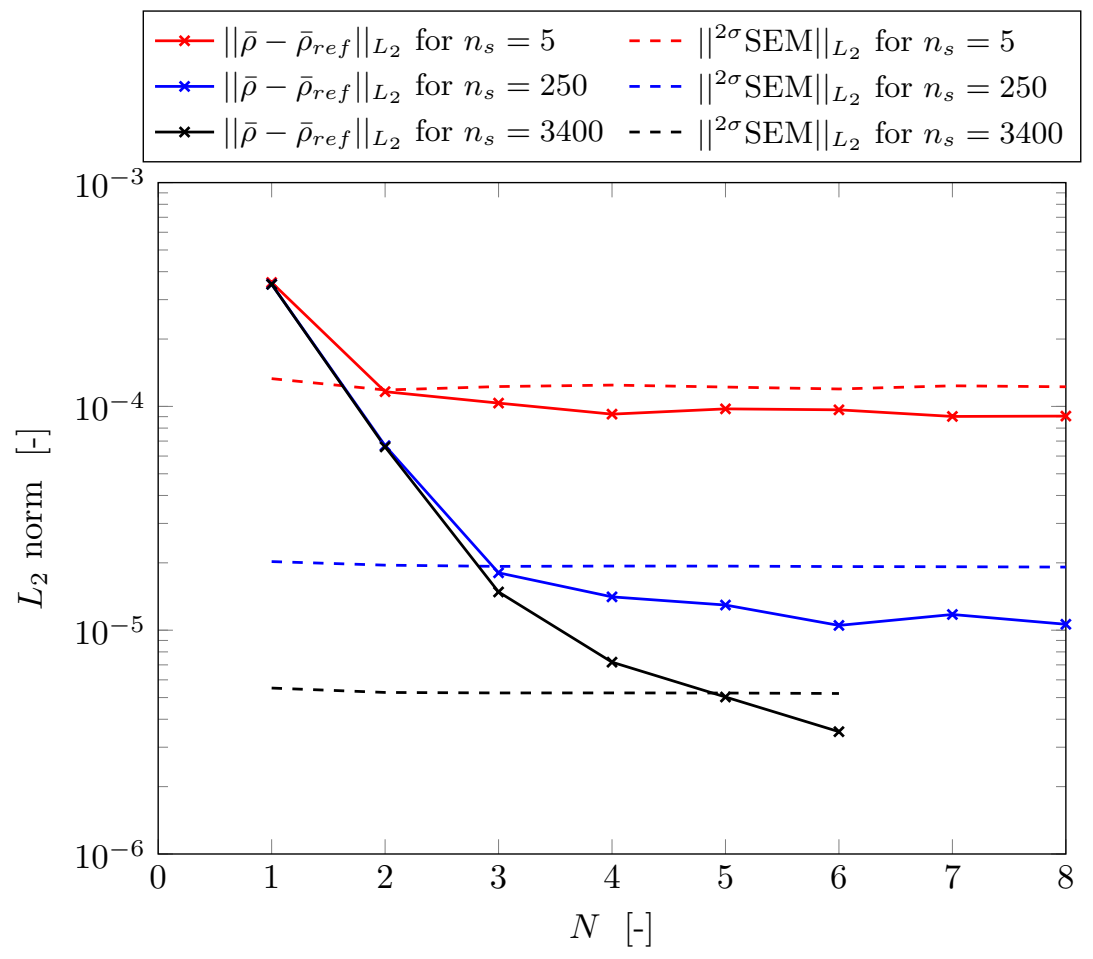

Figure 14: $L_{2}$ norm for domain 'A' with BR (reference $N_{\text {ref }}=7 ; 9 ; 9$ )

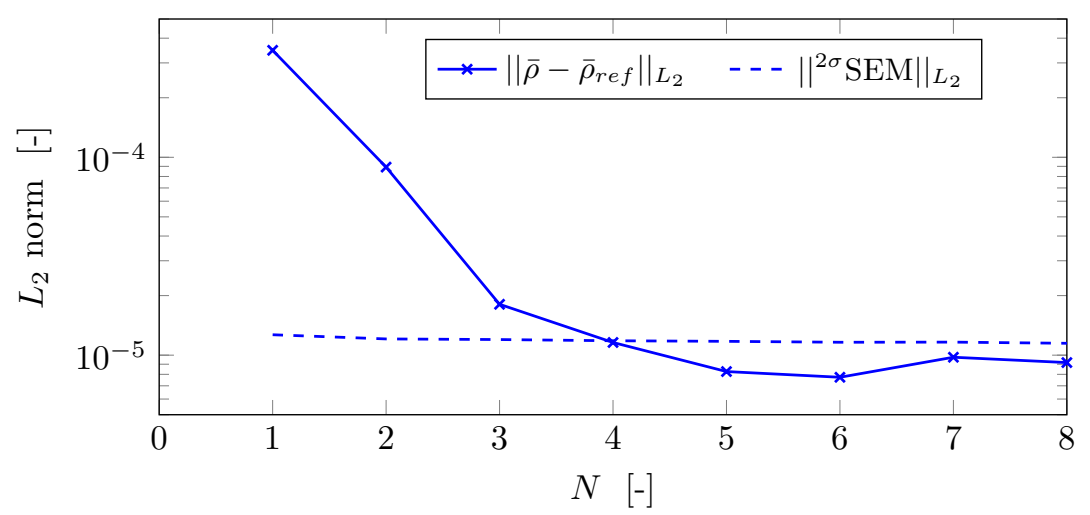

Figure 15: $L_{2}$ norm for domain 'B' with ions only $\left(n_{s}=250\right)$ 


\section{Conclusion}

An efficient Particle-in-Cell scheme based on a HDGSEM solver for electro-

290

is able to handle complex geometries by using unstructured curved meshes and also allows the simulation of jumps in permittivity from different dielectrical materials. Additionally, a Newton method is described to solve non-linear source terms arising from the Boltzmann fluid approximation for electrons. The hylytical solutions are known, including a dielectric sphere, a point source in a dielectric medium and a plasma sheath. The convergence of the field solver is investigated for different geometry approximations $\left(N_{\text {geo }}\right)$ and polynomial degrees $(N)$. It is shown that the expected convergence behaviour for increasing the geometry is used $\left(N \geq N_{\text {geo }}\right)$.

Finally, the applicability of the proposed method for complex electrostatic $3 \mathrm{D}$ problems is demonstrated with an ion optics simulation. Here, it is also shown how the error of the particle source in a PIC simulation is restricted

the particles, the accumulated error of the PIC simulation cannot drop under the statistical error due to the particle discretization independent of the $N$ and $N_{\text {geo }}$.

\section{Acknowledgements}

315

The authors gratefully thank the Deutsche Forschungsgemeinschaft (DFG) for funding this research within the projects "Kinetic Algorithms for the Maxwell- 
Boltzmann System and the Simulation of Magnetospheric Propulsion Systems" and "Coupled PIC-DSMC-Simulation of Laser Driven Ablative Gas Expansions". The latter being a sub project of the Collaborative Research Center 320 (SFB) 716 at the University of Stuttgart. The authors also like to thank Marco Restelli for his help in the derivation and implementation of the HDG solver. 
[1] T. Binder, M. Pfeiffer, S. Fasoulas, H. Leiter, High-fidelity particle-in-cell simulations of ion thruster optics, in: Proceedings of the 35th International Electric Propulsion Conference, ERPS, 2017.

[2] C. K. Birdsall, A. B. Langdon, Plasma Physics via Computer Simulation, Hilger, Bristol, 1991.

[3] C. K. Birdsall, Particle-in-cell charged-particle simulations, plus monte carlo collisions with neutral atoms, pic-mcc, IEEE Transactions on Plasma Science 19 (2) (1991) 65-85.

330

[4] R. W. Hockney, J. W. Eastwood, Computer Simulation Using Particles, Taylor \& Francis, 1988.

URL http://books .google.de/books?id=nTOFkmnCQuIC

n [5] J. Hesthaven, T. Warburton, Nodal Discontinuous Galerkin Methods: Algorithms, Analysis, and Applications, Texts in Applied Mathematics, Springer, 2008.

URL http://books.google.de/books?id=APQkDOmwyksC

๑ [6] D. A. Kopriva, Implementing Spectral Methods for Partial Differential Equations: Algorithms for Scientists and Engineers, Mathematics and Statistics, Springer, 2009. URL http://books.google.de/books?id=fZyqWPNjx4AC

[7] B. Cockburn, B. Dong, J. Guzmán, M. Restelli, R. Sacco, A hybridizable discontinuous Galerkin method for steady-state convection-diffusionreaction problems, SIAM Journal on Scientific Computing 31 (5) (2009) $3827-3846$.

[8] B. Cockburn, J. Gopalakrishnan, The derivation of hybridizable discontinuous galerkin methods for stokes flow, SIAM Journal on Numerical Analysis 47 (2) (2009) 1092-1125.

[9] B. Cockburn, J. Gopalakrishnan, New hybridization techniques, GAMMMitteilungen 28 (2) (2005) 154-182. 
[10] J. D. Jackson, Classical electrodynamics, 3rd Edition, Wiley, New York, NY, 1999.

[11] Y. Hu, J. Wang, Electron properties in collisionless mesothermal plasma expansion: fully kinetic simulations, IEEE Transactions on Plasma Science 43 (9) (2015) 2832-2838.

355 [12] I. D. Boyd, Review of hall thruster plume modeling, Journal of Spacecraft and Rockets 38 (3) (2001) 381-387.

[13] C.-D. Munz, M. Auweter-Kurtz, S. Fasoulas, A. Mirza, P. Ortwein, M. Pfeiffer, T. Stindl, Coupled Particle-In-Cell and Direct Simulation Monte Carlo method for simulating reactive plasma flows, Comptes Rendus Mécanique 342 (10-11) (2014) 662-670. doi:10.1016/j.crme.2014. 07.005

[14] G. B. Jacobs, J. S. Hesthaven, High-order Nodal Discontinuous Galerkin Particle-in-cell Method on Unstructured Grids, J. Comput. Phys. 214 (1) (2006) 96-121. doi:10.1016/j.jcp.2005.09.008. URL http://dx.doi.org/10.1016/j.jcp.2005.09.008

[15] B. Cockburn, B. Dong, J. Guzmán, M. Restelli, R. Sacco, A hybridizable discontinuous galerkin method for steady-state convection-diffusionreaction problems, SIAM J. Scientific Computing 31 (5) (2009) 3827-3846.

[16] F. Hindenlang, T. Bolemann, C.-D. Munz, Mesh Curving Techniques for High Order Discontinuous Galerkin Simulations, in: IDIHOM: Industrialization of High-Order Methods-A Top-Down Approach, Springer, 2015, pp. $133-152$.

[17] D. A. Kopriva, Metric identities and the discontinuous spectral element method on curvilinear meshes, Journal of Scientific Computing 26 (3) (2006) 301-327.

[18] A. Stock, J. Neudorfer, M. Riedlinger, G. Pirrung, G. Gassner, R. Schneider, S. Roller, C.-D. Munz, Three-Dimensional Numerical Simulation of a 
30-GHz Gyrotron Resonator With an Explicit High-Order DiscontinuousGalerkin-Based Parallel Particle-In-Cell Method, IEEE Transactions on Plasma Science 40 (7) (2012) 1860-1870.

[19] K.-U. Riemann, The bohm criterion and sheath formation, Journal of Physics D: Applied Physics 24 (4) (1991) 493.

[20] L. A. Schwager, C. K. Birdsall, Collector and source sheaths of a finite ion temperature plasma, Physics of Fluids B: Plasma Physics 2 (5) (1990) 1057-1068. 